\title{
Antimicrobial resistance in non-aureus staphylococci isolated from milk is associated with systemic but not intramammary administration of antimicrobials in dairy cattle
}

\author{
Diego B. Nobrega, ${ }^{*}{ }^{1}$ Jeroen De Buck, ${ }^{*} \dagger$ and Herman W. Barkema* ${ }^{*} \ddagger$ \\ *Department of Production Animal Health, Faculty of Veterinary Medicine, University of Calgary, 2500 University Dr., Calgary, AB, T2N 1N4, \\ Canada \\ †Canadian Bovine Mastitis and Milk Quality Research Network, St-Hyacinthe, QC, J2S 7C6, Canada \\ †Department of Reproduction, Obstetrics and Herd Health, Faculty of Veterinary Medicine, Ghent University, Salisburylaan 133,9820 Merelbeke, \\ Belgium
}

\begin{abstract}
Non-aureus staphylococci (NAS) are the bacteria most frequently isolated from bovine milk. Objectives of this study were to determine herd-level associations between antimicrobial use (AMU) and prevalence of antimicrobial resistance (AMR) and antimicrobial resistance genes in NAS according to antimicrobials and routes of administration. The AMR profile was determined using a micro-broth dilution method against a panel of 23 antimicrobials for 1,702 NAS isolates obtained from 89 herds. A subset of these isolates ( $\mathrm{n}=$ 405) was submitted to whole-genome sequencing, and the presence of AMR genes was determined using data from 4 databases. Antimicrobial use was determined for all herds using an inventory of empty drug containers and quantified for each antimicrobial as the number of antimicrobial daily doses administered. Generalized linear models were used to estimate antimicrobial and route-specific associations between AMR in NAS and AMU. Prevalence of multidrug resistance in NAS was associated with systemic use of antimicrobials. Estimated relative risk associated with a 1-unit increase in antimicrobial daily doses per cow-year administered systemically was 1.28 . No association was present with either intramammary or intrauterine use. Three drug classes, all of high or very high importance for human medicine, were associated with drug-specific AMR when administered systemically: penicillins, thirdgeneration cephalosporins, and macrolides. Prevalence of tet, erm, and $b l a_{\mathrm{ARL}}$ genes in NAS was higher in herds that used more tetracyclines, macrolides, and third-generation cephalosporins, respectively. No association between drug-specific AMU and prevalence
\end{abstract}

Received February 5, 2018.

Accepted March 25, 2018.

${ }^{1}$ Corresponding author: diego.nobrega@ucalgary.ca of blaZ, $m p h C$, and $m s r A$ was identified, irrespective of route of administration. The either weak or nonexistent association between AMR and antimicrobials administered intramammarily suggest that a decrease in AMR of NAS following implementation of selective dry cow therapy would be minimal in comparison to reduced use of systemic antimicrobials.

Key words: antimicrobial use, antimicrobial resistance, non-aureus staphylococci, route of administration

\section{INTRODUCTION}

Antimicrobial resistance (AMR) has emerged as one of the most important threats to human and animal health globally. Without urgent action, it is estimated that 10 million human deaths per year will be attributable to AMR by 2050 (O'Neill, 2016). Because antimicrobial therapy is a key factor for emergence of AMR (Davies and Davies, 2010), recent efforts to mitigate the rapid rise of AMR have emphasized reduced use of antimicrobials. Indeed, it was recently reported that restricted use of antimicrobials in food-producing animals was associated with a decreased prevalence of AMR in these animals (Tang et al., 2017).

Bovine mastitis is the primary reason for antimicrobial use (AMU) on dairy farms worldwide (Pol and Ruegg, 2007; Saini et al., 2012a; Nobrega et al., 2017). Although it is well established that indiscriminate and poorly conducted treatments are responsible for emergence and amplification of AMR in veterinary medicine (Locatelli et al., 2010), limited evidence supports an increase in AMR in mastitis pathogens as a result of well-conducted treatments in dairy herds (Erskine et al., 2002). Due to intense pressure to reduce overall use of antimicrobials in food-producing animals, use of selective dry cow therapy (DCT), a very effective method for decreasing AMU in dairy herds, is being considered over blanket DCT in North America (Barkema et al., 2015; Kromker and Leimbach, 2017). This 
has been implemented in Scandinavia for decades and was recently successfully implemented in the Netherlands (Lam et al., 2017).

The overall effect of antimicrobial therapy on the emergence of AMR in bovine mastitis pathogens depends on several factors, including levels of antimicrobials in the mammary gland (e.g., pharmacokinetics) and causative microorganism (e.g., pharmacodynamics). Route of administration, dosage, frequency, duration, and number of treatments directly affect the quantity of antimicrobials that reach the udder, as well as extent and duration of bacterial exposure. Similar to AMR in gut microbiota (Zhang et al., 2013), associations between AMU and AMR in bovine mastitis pathogens may depend on the type of antimicrobial and route of administration.

Non-aureus staphylococci (NAS) are the most frequently isolated bacteria from bovine mastitis worldwide (De Visscher et al., 2017). Due to the high prevalence of these bacteria in the udder, they are in frequent contact with antimicrobials administered to dairy cows. Consequently, insights could be gained about associations between AMU and AMR in mastitis pathogens by monitoring the AMR profile of NAS isolates obtained from herds with varying levels and strategies of AMU. Therefore, the overall objective of this study was to evaluate the association between AMU and prevalence of AMR in NAS isolated from milk in Canadian dairy herds. Specific objectives were to (1) determine herdlevel associations between AMU and prevalence of AMR in NAS, and (2) verify whether associations are specific for antimicrobials or routes of administration.

\section{MATERIALS AND METHODS}

\section{Herds and Isolates}

Participating herds and bacteria identification protocol have been described (Reyher et al., 2011). Briefly, 89 herds selected to represent their respective provinces in terms of bulk tank SCC, housing type, breed, and milking schedule were monitored for approximately $2 \mathrm{yr}$ (from February 2007 to December 2008). Quarter milk samples were collected systematically from lactating cows using 3 protocols: (1) clinical mastitis (CM) samples, (2) samples from cows before and after drying-off, and (3) samples from randomly selected cows. Overall, 115,294 milk samples were obtained longitudinally from 5,157 lactating cows. Following bacteriological culture and identification performed according to standardized guidelines (NMC, 1999), NAS were obtained from 5,149 milk samples where IMI was defined as $\geq 1,000$ NAS cfu/mL of milk in pure culture. The NAS isolates had been previously identified to the species level us- ing partial sequencing of the rроB gene (Condas et al., 2017). In addition, NAS-positive samples were obtained at least once from 2,091 cows, with 5,094 isolates from 1,702 cows available for the present study. From this total, a random selection of a single NAS isolate per cow, irrespective of species and sampling set, was done, resulting in 57 NAS isolates from $\mathrm{CM}$ and 1,645 NAS isolates from nonclinical IMI obtained from 1,702 cows.

A subset of these isolates $(n=405)$ obtained from 87 herds was submitted to whole-genome sequencing using a MiSeq platform, following sample preparation using the Nextera XT DNA Library Prep kit from Illumina (Naushad et al., 2016). The selection protocol favored inclusion of samples from CM and NAS of uncommon species, followed by random selection of a subset of isolates for the 7 most common species (Staphylococcus chromogenes, S. simulans, S. xylosus, S. haemolyticus, S. epidermidis, S. cohnii, and S. sciuri) and also isolates with a multidrug-resistant (MDR) phenotype (Nobrega et al., 2018).

\section{AMR Profile}

Phenotypic AMR profile was determined for all isolates using the micro-broth dilution method against a panel of 23 antimicrobials, following standardized guidelines (CLSI, 2016). Antimicrobials and range of concentrations evaluated were ampicillin $(0.12-8 \mu \mathrm{g} /$ $\mathrm{mL})$, chloramphenicol $(2-16 \mu \mathrm{g} / \mathrm{mL})$, ceftiofur $(0.5-4$ $\mu \mathrm{g} / \mathrm{mL})$, cephalotin $(2-16 \mu \mathrm{g} / \mathrm{mL})$, ciprofloxacin (1-2 $\mu \mathrm{g} / \mathrm{mL})$, clindamycin $(0.5-2 \mu \mathrm{g} / \mathrm{mL})$, daptomycin (0.5-4 $\mu \mathrm{g} / \mathrm{mL})$, erythromycin $(0.25-4 \mu \mathrm{g} / \mathrm{mL})$, gentamicin $(2-16 \mu \mathrm{g} / \mathrm{mL})$, levofloxacin $(0.25-4 \mu \mathrm{g} / \mathrm{mL})$, linezolid $(1-8 \mu \mathrm{g} / \mathrm{mL})$, moxifloxacin $(0.25-4 \mu \mathrm{g} / \mathrm{mL})$, nitrofurantoin $(32-64 \mu \mathrm{g} / \mathrm{mL})$, oxacillin $+2 \% \mathrm{NaCl}$ $(0.25-4 \mu \mathrm{g} / \mathrm{mL})$, penicillin $(0.06-8 \mu \mathrm{g} / \mathrm{mL})$, penicillin/ novobiocin $(1 / 2-8 / 16 \mu \mathrm{g} / \mathrm{mL})$, pirlimycin $(0.5-4 \mu \mathrm{g} /$ $\mathrm{mL})$, quinupristin/dalfopristin $(0.5-4 \mu \mathrm{g} / \mathrm{mL})$, rifampin $(0.5-4 \mu \mathrm{g} / \mathrm{mL})$, tetracycline $(2-16 \mu \mathrm{g} / \mathrm{mL})$, tigecycline $(0.03-0.5 \mu \mathrm{g} / \mathrm{mL})$, trimethoprim/sulfamethoxazole $(0.05 / 9.5-4 / 76 \mu \mathrm{g} / \mathrm{mL})$, and vancomycin $(0.25-32 \mu \mathrm{g} /$ $\mathrm{mL}$ ). Staphylococcus aureus ATCC 29213 was used as a quality control strain for all reactions. Minimum inhibitory concentration breakpoints were defined according to the CLSI criteria for Staphylococcus spp. (CLSI, 2013, 2016) with minor modifications (S. aureus breakpoint was used for ceftiofur, oxacillin, penicillin/novobiocin, and pirlamycin). Animal criteria were used if available. Tigecycline breakpoint was based on EUCAST guidelines (EUCAST, 2017). Isolates were classified as either susceptible or resistant, with MIC values equal or higher than the intermediate breakpoint (when available) considered resistant. Multidrug resistance was defined as resistance against $\geq 2$ antimicrobial classes. 
Presence of AMR genes (ARG) was determined using data from 4 databases: (1) ARG-ANNOT v3 (Antibiotic Resistance Gene-ANNOTation; Gupta et al., 2014); (2) MegaRES v1.0.1 (Lakin et al., 2017); (3) Comprehensive Antibiotic Resistance Database v1.1.6 (CARD; Jia et al., 2017); and (4) ResFinder from the Center for Genomic Epidemiology (as of November 2, 2016; Zankari et al., 2012). Databases were first combined into a single nonredundant list of queries, and a personalized BLASTp server was used to perform local alignments against sequenced genomes. A single best hit per query per isolate, defined as one with highest product between query coverage and pairwise identity, was initially obtained. Hits obtained for the same loci in a genome were then compared, and a single best hit was retained based on the same metric. Finally, host sequences were used as a query against the National Center for Biotechnology Information nonredundant database $(n r)$ to confirm presence of the respective ARG. A threshold of $\geq 80 \%$ pairwise identity and query coverage was used for confirmation. For further analyses, only ARG known to be associated with AMR in NAS (Nobrega et al., 2018) were considered, including the mphC, erm (ermA, ermC, ermT), msrA, tet (tetK, tet $L$, tet $M)$, mecA, fexA, bla $a_{\mathrm{ARL}}$, and blaZ genes.

\section{Antimicrobial Use}

Antimicrobial use data collection has been described (Saini et al., 2012a). In brief, farm personnel were instructed by veterinarians and technicians to dispose of all empty drug containers into 40-L receptacles, strategically placed on farms for approximately $2 \mathrm{yr}$ (from February 2007 to December 2008). Farms were visited monthly by students and technicians, and number of empty containers as well as detailed information including date, antimicrobial, and weight or volume of the container were recorded. Data were entered into spreadsheets (Microsoft Excel, Microsoft Corp., Redmond, WA). The number of expected antimicrobial daily doses (ADD) was estimated based on weight of active antimicrobial ingredient per container, recommended on-label daily dosage, and animal BW. A preliminary assessment using on-farm treatment records was done to verify the animal category (young stock; $<1$ yr old, or standard adult dairy cow; $>1$ yr old) most likely to be treated with each antimicrobial (Nobrega et al., 2017). For antimicrobials commonly administered to young stock, a standard BW of $200 \mathrm{~kg}$ per animal was used. Otherwise, ADD were estimated considering a 600-kg adult dairy cow (Jensen et al., 2004). The ADD were standardized into antimicrobial drug use rates (ADUR; ADD/cow-year), a time-sensitive metric of antimicrobial consumption not affected by total herd size, length of follow-up, or both. Antimicrobials were classified according to route of administration into systemic, intramammary, or intrauterine, and according to class [penicillins, penicillin combinations (combination of penicillin and novobiocin, and combination containing penicillin, novobiocin, streptomycin, and polymyxin B), first- and third-generation cephalosporins, combination of sulfonamides and trimethoprim, tetracyclines, lincosamides, phenicols, and macrolides].

\section{Statistical Analyses}

Analyses were done at the herd level. Herd-level prevalence of resistant isolates was defined as the number of resistant NAS isolates, divided by number of NAS isolates tested (or number of NAS-positive cows included per herd). Generalized linear models were fit using maximum likelihood, with herd-level number of resistant isolates as the outcome, assumed to follow a negative binomial distribution, and offset defined as the logarithm of the number of isolates tested per herd. For all analyses, the log link was used for modeling expected values. To estimate the association between AMU and AMR, AMU defined as herd-level ADUR irrespective of class and route of administration was considered the predictor, whereas number of MDR NAS was considered the outcome. Antimicrobial-specific associations were then evaluated individually, where resistance to each antimicrobial class was modeled as a function of drug-specific ADUR. Antimicrobials evaluated were penicillin, penicillin combinations, first-generation cephalosporins, third-generation cephalosporins, tetracyclines, lincosamides, phenicols, and macrolides.

Effect of route of administration was evaluated by considering route-specific ADUR into previous analyses, as well as all possible 2- and 3-way interactions between intramammary, systemic, and intrauterine ADUR. Prior to analyses, route-specific ADUR for each drug class was summarized. If $<5$ herds had been given an antimicrobial by a specific route, the term was removed before analyses. For example, no intrauterine use of $\beta$-lactams was recorded. In that instance, intrauterine ADUR of penicillins was removed from further analyses, and log of the expected number of $\beta$-lactamresistant NAS (ampicillin, ceftiofur, cephalotin, oxacillin, penicillin, and its combinations) was modeled as a function of systemic, intramammary ADUR of penicillins, as well as their interaction. Terms were removed from models after careful modification and confounding assessment, where interaction $P$-values and relative changes of $\geq 15 \%$ of remaining predictors were considered, respectively.

Antimicrobials and routes associated with drugspecific AMR at $P<0.20$ were considered for further 
analyses, where number of MDR isolates was considered as the outcome and ADUR of antimicrobials associated with AMR were predictors. Two-way interactions between antimicrobials, where the effect of a particular drug class may depend on a second drug class being used or not, were also considered at this stage. Predictors were removed one at a time according to $P$-values and confounding assessments, as described above. Antimicrobials and routes not selected in the initial screening were then added one at a time to verify their relationship with AMR, conditional on the presence of selected model terms. The same approach was used for $\beta$-lactams exclusively, where antimicrobials and routes associated with $\beta$-lactam resistance at $P<0.20$ were considered in the same analysis.

Associations between ARG observed at least once in $\geq 10$ herds (blaZ, bla $a_{\mathrm{ARL}}$, erm, mphC, msrA, and tet) and drug-specific use were determined in individual Poisson models. For these analyses, herd-level number of ARG was considered as the outcome, assumed to follow a Poisson distribution with offset defined as the logarithm of number of isolates sequenced per herd. The log link was used for modeling the expected values. The dispersion parameter $(\phi)$ was estimated from the data and used in analyses to account for possible overdispersion. Systemic and intramammary ADUR, as well as their interaction, were considered predictors. Terms were removed from models one at a time following modification and confounding assessment, as described above.

For all analyses, the assumption of linearity was deemed tenable after visual inspection of scatterplots, where the log of the herd-level proportion of resistant isolates was plotted against categories of ADUR, and LOWESS curves were visualized. Statistical significance was considered as $P<0.05$. The $P$-values from final models were adjusted for multiple comparisons by controlling the false discovery rate using the p.adjust function in R (Benjamini and Hochberg, 1995). Analyses were repeated using 2 approaches: (1) overdispersed binomial regression models, where number of isolates per herd was considered as number of trials; and (2) $\beta$-regression models, where herd-level proportion of resistance was used as an outcome, and also excluding isolates from $\mathrm{CM}$ and MDR isolates sequenced on purpose. Findings were not dependent on either method of analysis or presence of additional isolates; therefore, results from the initial approach were presented as relative risks (RR) and 95\% confidence intervals. For visual presentation of results, boxplots were produced using total and route-specific ADUR as categories. All analyses were done in $\mathrm{R}$ version 3.4.1 ( $\mathrm{R}$ Core Team, 2017) using the packages betareg (Cribari-Neto and
Zeileis, 2010), MASS (Venables and Ripley, 2002), and stats.

Assuming a prevalence of MDR in NAS isolates of 0.02 (Nobrega et al., 2018), an average of 19 isolates was included per herd, and a herd-average of 1.7 ADD per cow-year (Nobrega et al., 2017), this study had a power of $93 \%$ to detect a RR of 1.5 associated with an unit increase in ADD per cow-year using overdispersed binomial regression models under simulation. Similarly, a power of $80 \%$ was expected to detect a RR of 1.4 under the same conditions. As for sequenced isolates, assuming a prevalence of ARG of $0.01,5$ isolates sequenced per herd on average, and 1.7 ADD per cowyear on average, $80 \%$ power was expected to detect a $\mathrm{RR}$ of 2 associated with a 1-unit increase in the number of ADD per cow-year.

\section{RESULTS}

\section{$A M R$}

On average, 19 NAS isolates and 4.7 sequenced isolates were included per herd (ranges, 4 to 41 and 1 to 13, respectively). Resistance was commonly observed against tetracycline (206 isolates; $12 \%$ ), penicillin (204 isolates; $12 \%$ ), erythromycin (141 isolates; $8 \%$ ), pirlimycin (111 isolates; 7\%), chloramphenicol (77 isolates; $5 \%$ ), and clindamycin (68 isolates; $4 \%$ of isolates). In contrast, no NAS isolate was resistant to gentamicin, linezolid, cephalotin, vancomycin, levofloxacin, moxifloxacin, ciprofloxacin, or nitrofurantoin. Twenty-three isolates $(1.4 \%)$ were resistant to the combination of penicillin and novobiocin. Five $(0.3 \%)$ NAS isolates were resistant to the combination of trimethoprim and sulfamethoxazole. Five isolates were resistant to daptomycin. A single isolate was resistant to rifampin. Five isolates were resistant to ceftiofur; 1 of these 5 isolates was also resistant to oxacillin. A total of 150 isolates (8.8\%) had a MDR phenotype (resistant against $\geq 2$ drug classes). Mean herd-level prevalence of MDR in NAS-positive cows was $8 \%$, ranging from 0 to $44 \%$.

The $m p h C$ gene was present in $74(18 \%)$ isolates, whereas other macrolide-resistance determinants such as erm genes and the $m s r A$ were present in $12(3 \%)$ and $42(10 \%)$ isolates, respectively. In addition, tet genes were present in $63(16 \%)$ isolates. The mecA resistance determinant and the chloramphenicol/florfenicol efflux MFS transporter FexA represented by the fexA gene were present in 4 and 5 NAS isolates, respectively. Furthermore, blaZ was detected in 42 isolates (10\%), being the most common $\beta$-lactam resistance determinant detected, whereas the $b l a_{\mathrm{ARL}}$ gene was present in all 14 (3\%) Staphylococcs arlettae isolates sequenced. 


\section{$A M U$}

Eighty herds recorded at least 1 ADD using penicillins (Table 1). A total of 85, 76, and 80 herds recorded at least 1 ADD of penicillin combinations, first- and third-generation cephalosporins, respectively. A combination of sulfonamides and trimethoprim was used at least once in 68 herds, whereas 52,31 , and 29 herds recorded at least 1 treatment using lincosamides, macrolides, and phenicols, respectively. Average herd-level ADUR was 3.7, ranging from 0.7 to 14.4 ADD per cowyear. Antimicrobials commonly used included penicillins, combinations including penicillins, first- and thirdgeneration cephalosporins, tetracyclines, combinations of sulfonamides and trimethoprim, and lincosamides (Table 1). In contrast, macrolides (0.07 ADD per cowyear on average), phenicols (0.03 ADD per cow-year on average), aminoglycosides (0.004 ADD per cow-year on average), and fluoroquinolones (0.004 ADD per cowyear on average) were rarely used as treatment in these dairy herds. Third-generation cephalosporins, penicillins, combinations of sulfonamides and trimethoprim, and tetracyclines were commonly administered systemically, whereas penicillins and their combinations, first-generation cephalosporins, and lincosamides were the most commonly used intramammary antimicrobial agents (Table 1). Of note, penicillin combinations, lincosamides, and first-generation cephalosporins were used almost exclusively by the intramammary route (Table 1).

\section{Association Between AMU and AMR}

Total AMU, defined as ADUR (number of ADD per cow-year), was positively associated with prevalence of MDR in NAS (Figure 1). The estimated RR associated with an increase of 1 ADD per cow-year was 1.09 (95\% CI: 1.00-1.18; Table 2; Figure 3). Only systemic use was associated with prevalence of MDR in NAS isolates (Figures 2 and 3), whereas intramammary and intrauterine ADUR were not. Estimated RR associated with an unit increase in ADD per cow-year administered systemically was 1.28 (Figure 3). Hence, prevalence of MDR in NAS was $28 \%$ higher for herds that administered systemically 2 ADD per cow-year than the same for herds that administered a single ADD per cow-year.

Systemic use of penicillins, macrolides, and thirdgeneration cephalosporins was associated with prevalence of resistance against that specific drug class in separate analyses. Associations detected were neither confounded nor modified by intramammary use of the same antimicrobials, which were not associated with prevalence of resistance in NAS isolates (Table 2). Estimated RR associated with an increase of 1 ADD per cow-year were $1.55,1.36$, and 4.81 for penicillins, thirdgeneration cephalosporins, and macrolides, respectively (Table 2; Figure 3). The ADUR of tetracyclines, penicillins combination, lincosamides, and first-generation cephalosporins were not associated with drug-specific AMR in NAS, irrespective of route of administration.

When considering effects of systemic penicillins, macrolides, and third-generation cephalosporins on prevalence of MDR in NAS, associations were detected only for macrolides and third-generation cephalosporins. No significant 2-way interaction between antimicrobial classes was present; therefore, observed effects did not depend on systemic use of a second antimicrobial. Estimated RR of MDR in NAS associated with an increase of an ADD per cow-year were 1.36 (95\% CI: 0.87-2.12), 1.36 (95\% CI: $1.04-1.76)$, and 2.93 (95\% CI: 1.24-6.93) for penicillins, third-generation cephalosporins, and macrolides, respectively. When considering ef-

Table 1. Antimicrobial use of dairy herds recording at least 1 antimicrobial daily dose by route of administration and antimicrobial of choice

\begin{tabular}{|c|c|c|c|c|c|c|c|c|}
\hline \multirow[b]{3}{*}{ Antimicrobial } & \multicolumn{6}{|c|}{ Route of administration } & & \\
\hline & \multicolumn{2}{|c|}{ Intramammary } & \multicolumn{2}{|c|}{ Systemic } & \multicolumn{2}{|c|}{ Intrauterine } & \multicolumn{2}{|c|}{ All } \\
\hline & No. ${ }^{1}$ & $\mathrm{ADUR}^{2}$ & No. & ADUR & No. & ADUR & No. & ADUR \\
\hline All & 87 & 1.90 & 88 & 1.79 & 19 & 0.01 & 89 & 3.70 \\
\hline Penicillin combinations & 85 & 1.09 & - & - & - & - & 85 & 1.09 \\
\hline Third-generation cephalosporins & 28 & 0.04 & 77 & 0.63 & - & - & 80 & 0.67 \\
\hline Penicillins & 51 & 0.17 & 75 & 0.48 & - & - & 80 & 0.65 \\
\hline First-generation cephalosporins & 73 & 0.35 & - & - & 14 & 0.004 & 76 & 0.35 \\
\hline Sulfonamide combinations & - & - & 68 & 0.34 & - & - & 68 & 0.34 \\
\hline Tetracyclines & - & - & 56 & 0.24 & 7 & 0.007 & 57 & 0.25 \\
\hline Lincosamides & 52 & 0.25 & - & - & - & - & 52 & 0.25 \\
\hline Phenicols & - & - & 29 & 0.03 & - & - & 29 & 0.03 \\
\hline Macrolides & 4 & 0.003 & 27 & 0.07 & - & - & 31 & 0.07 \\
\hline
\end{tabular}

${ }^{1}$ Number of herds that used the antimicrobial drug class at least once.

${ }^{2}$ Antimicrobial daily doses per cow-year. 


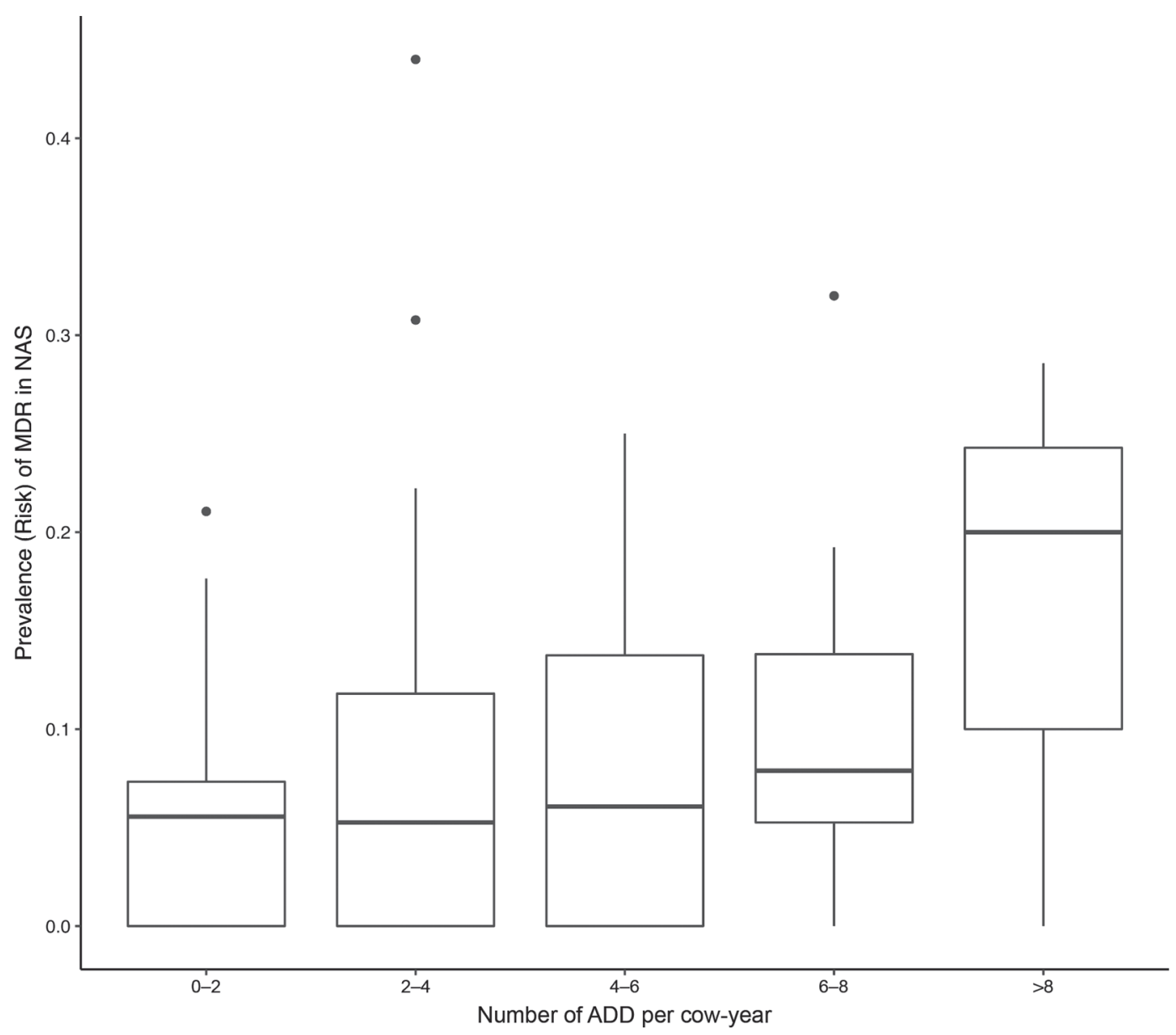

Figure 1. Herd-level prevalence of multidrug resistance (MDR) against $\geq 2$ antimicrobial classes in non-aureus staphylococci (NAS) isolates by number of antimicrobial daily doses (ADD) per cow-year. The box shows the lower quartile, median, and upper quartile of the herd-level prevalence of MDR. Whiskers show the upper and lower adjacent values (defined as values within 1.5 times the interquartile range from the upper and lower quartile, respectively). Circles are considered outliers.

fects of penicillins and third-generation cephalosporins administered systemically on prevalence of $\beta$-lactam resistance in NAS, estimated $\mathrm{RR}$ associated with an increase of an ADD per cow-year were 1.53 (95\% CI: 1.06-2.20) and 1.28 (95\% CI: $1.03-1.59)$ for penicillins and third-generation cephalosporins, respectively.

Prevalence of erm genes in NAS was 10.63 (95\% CI: 2.79-40.59) times higher for each increase of 1 ADD of macrolides per cow-year administered systemically. Prevalence of tet genes in NAS was 2.41 (95\% CI: 1.42-4.08) times higher for each increase of 1 ADD of tetracyclines per cow-year administered systemically. Similarly, systemic use of third-generation cephalosporins was associated with prevalence of the $b l a_{\mathrm{ARL}}$ gene in NAS (RR $=1.95 ; 95 \%$ CI: 1.08-3.52). No association between drug-specific AMU and prevalence of blaZ, $m p h C$, and $m s r A$ was present, irrespective of route of administration (Table 3 ).

\section{DISCUSSION}

Prevalence of AMR in NAS was associated with systemic use of antimicrobials in dairy herds. In contrast, no association was detected with neither intramammary nor intrauterine use of antimicrobials. Three drug classes were associated with drug-specific AMR when administered systemically: penicillins, third-generation cephalosporins, and macrolides. Prevalence of tet, erm, and $b l a_{\mathrm{ARL}}$ genes was higher in NAS for herds that used more tetracyclines, macrolides, and third-generation cephalosporins, respectively.

The 3 drug classes associated with drug-specific AMR (third-generation cephalosporins, penicillins, and macrolides) are considered critically important antimicrobials for human medicine. Two of them (third-generation cephalosporins and macrolides) are among antimicrobials of highest priority, according to the World Health 


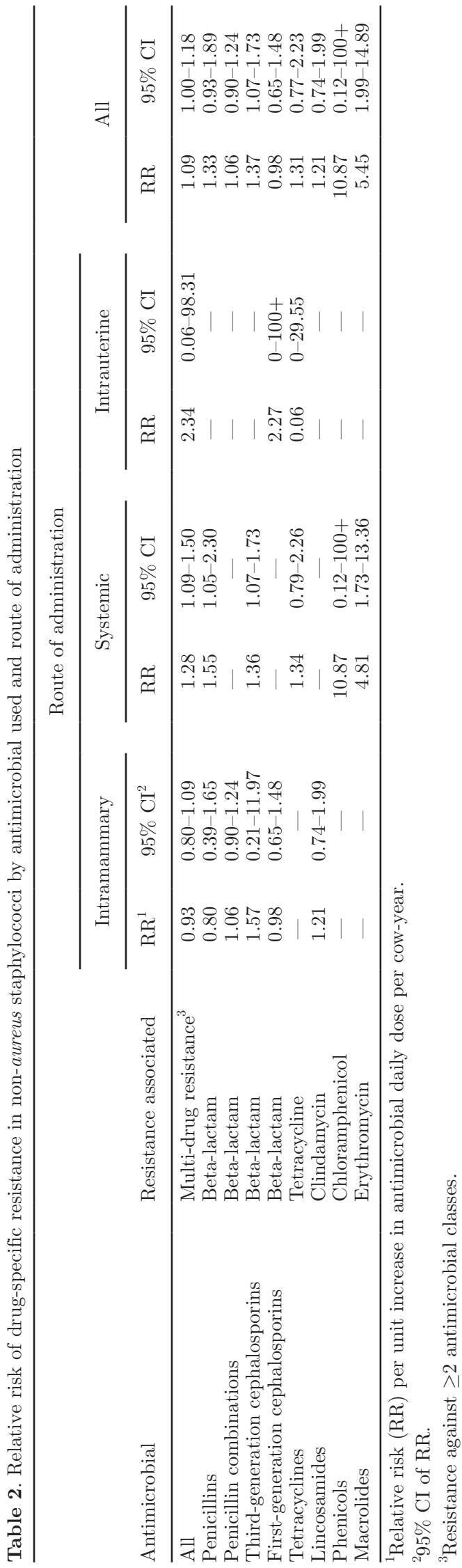

Organization (WHO, 2016), meaning that their use in veterinary medicine should be limited. Although it is not known whether NAS of bovine origin pose a risk to human health, the precautionary principle is warranted, especially because interspecies transfer is well

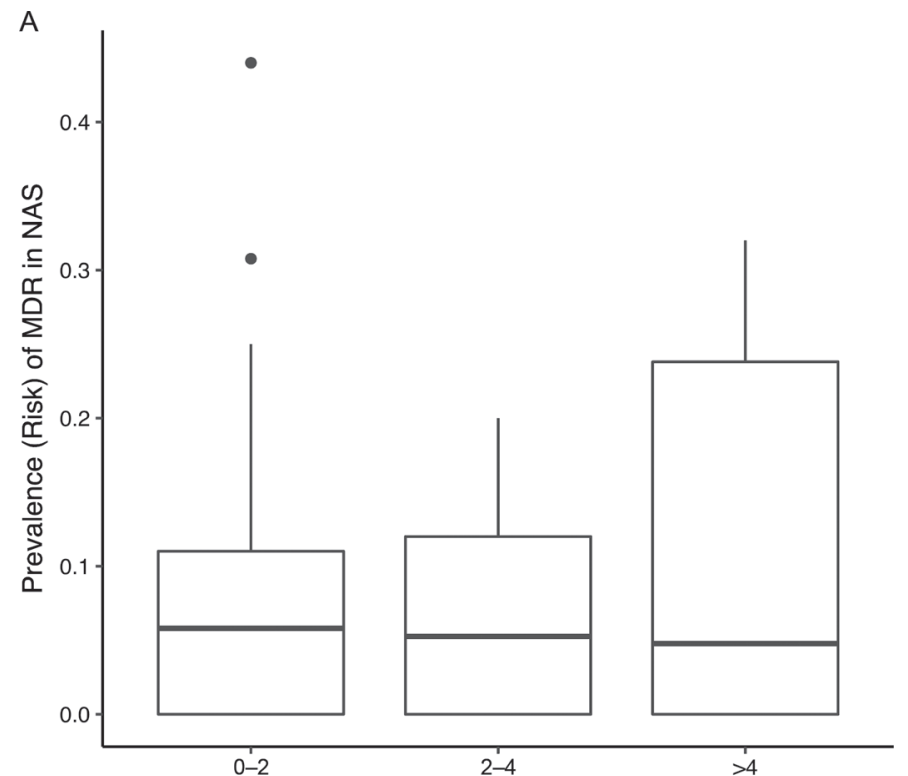

B

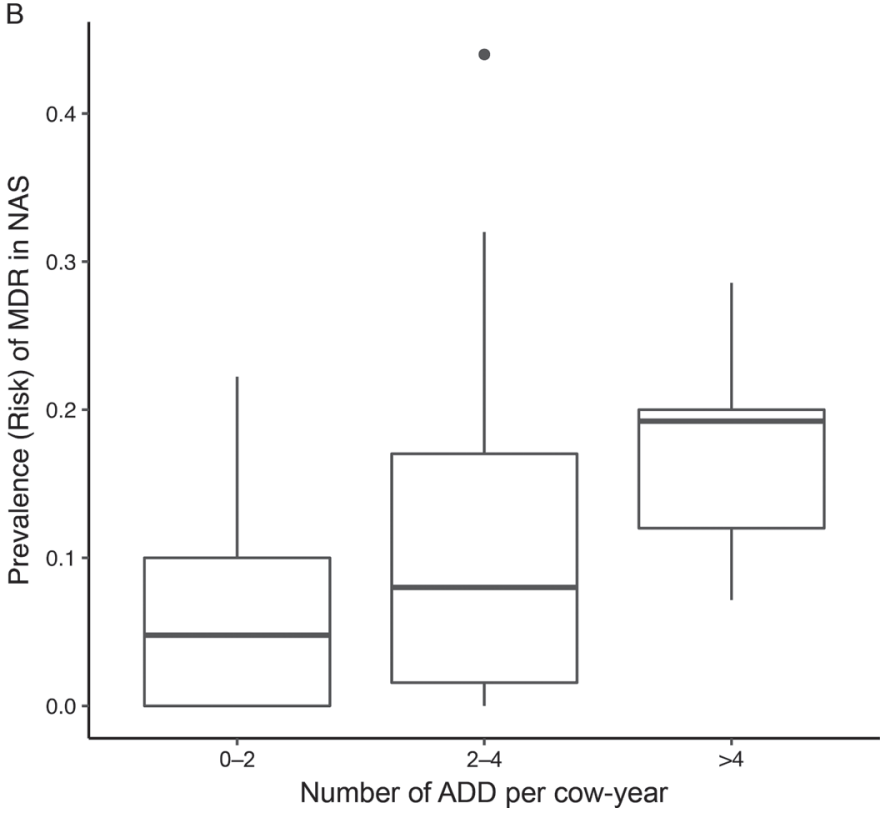

Figure 2. Herd-level prevalence of multidrug resistance (MDR) against $\geq 2$ antimicrobial classes in non-aureus staphylococci (NAS) isolates by number of intramammary (A) and systemic (B) antimicrobial daily doses (ADD) per cow-year. The box shows the lower quartile, median, and upper quartile of the herd-level prevalence of MDR. Whiskers show the upper and lower adjacent values (defined as values within 1.5 times the interquartile range from the upper and lower quartile, respectively). Circles are considered outliers. 
documented (Thorberg et al., 2006), and NAS may act as important reservoirs of ARG.

Concentrations of antimicrobials in the udder and other sites after systemic administration depend on lipid solubility, ionization, route of administration, plasma protein binding, and chemical nature of the drug. For any antimicrobial therapy, it is important that drug concentration exceeds the minimum concentration required to prevent resistance amplification (e.g., concentration termed as mutant prevention concentra- tion) for a sufficient duration (Blondeau, 2009). We hypothesize that associations between AMU and AMR in NAS for antimicrobials administered systemically were a consequence of prolonged bacterial exposure to sub-therapeutic antimicrobial concentrations in the udder and especially other tissues following systemic treatments not intended for IMI, a process analogous to fecal carriage of resistant bacteria after treatment for urinary tract infections in humans (Jorgensen et al., 2017). The NAS are (partially) commensal bacteria,
Antimicrobial

\section{All routes}

All

Penicillins

Penicillin combinations

3rd generation cephalosporins

1st generation cephalosporins

Tetracyclines

Lincosamides

Phenicols

Macrolides

\section{Intramammary}

\section{All}

Penicillins

Penicillin combinations

3rd generation cephalosporins

1 st generation cephalosporins

Tetracyclines

Lincosamides

Phenicols

Macrolides

\section{Systemic}

All

Penicillins

Penicillin combinations

3rd generation cephalosporins

1st generation cephalosporins

Tetracyclines

Lincosamides

Phenicols

Macrolides
Resistance

Relative risk $[95 \% \mathrm{Cl}]$

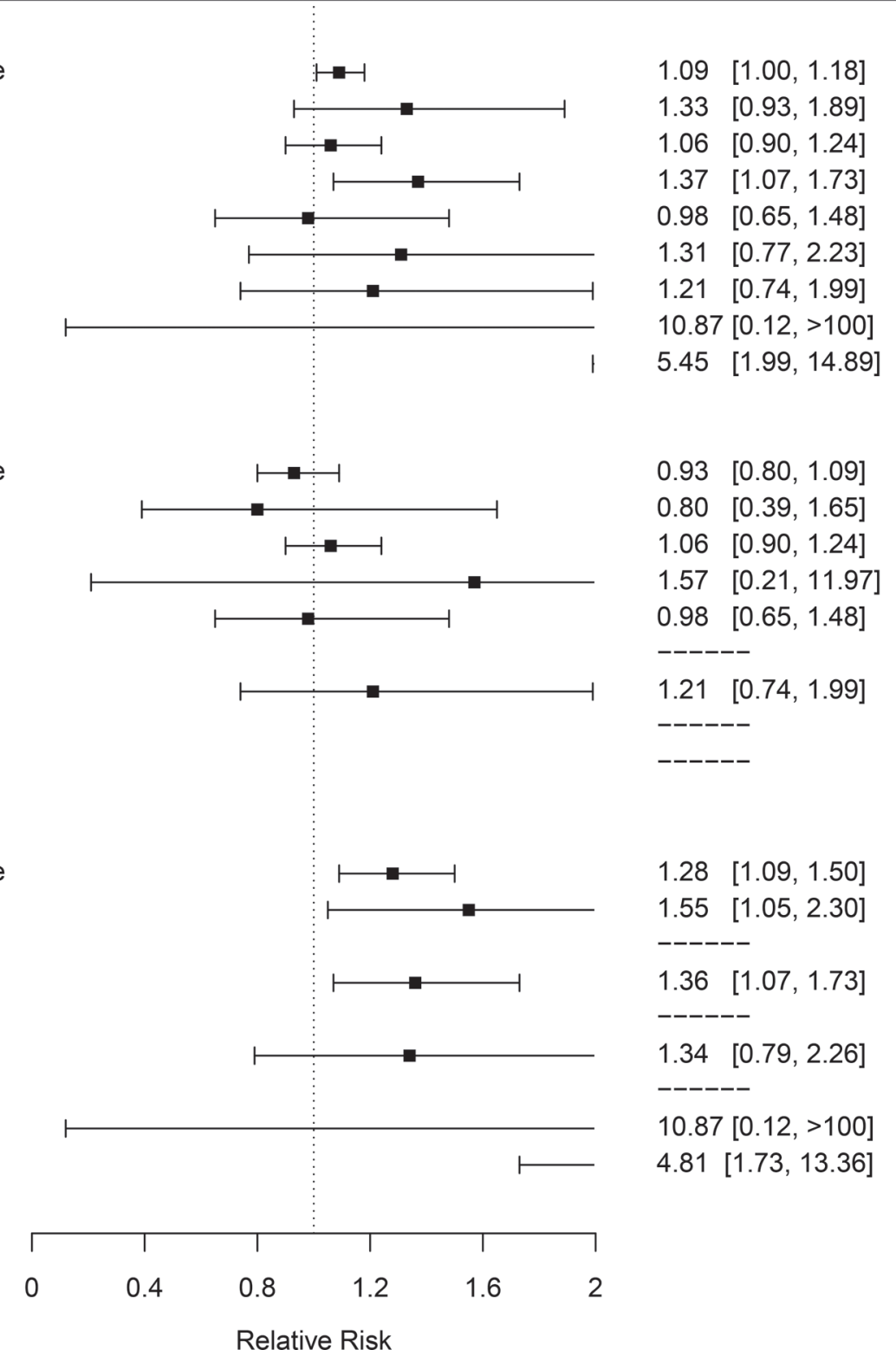

Multidrug resistance

Beta-lactams

Beta-lactams

Beta-lactams

Beta-lactams

Tetracycline

Clindamycin

Chloramphenicol

Erythromycin

$1.09[1.00,1.18]$

$1.33[0.93,1.89]$

$1.06[0.90,1.24]$

$1.37[1.07,1.73]$

$.98[0.65,1.48]$

$.31[0.77,2.23]$

$21 \quad[0.74,1.99]$

$0.87[0.12,>100]$

Chloramphenicol

Multidrug resistance

Beta-lactams

Beta-lactams

Beta-lactams

Beta-lactams

Tetracycline

Clindamycin

Chloramphenicol

Erythromycin

$1.28 \quad[1.09,1.50]$

$1.55[1.05,2.30]$

$1.36[1.07,1.73]$

$1.34[0.79,2.26]$

$10.87[0.12,>100]$

$4.81[1.73,13.36]$

Figure 3. Relative risks (RR) and $95 \% \mathrm{CI}$ associated with an unit increase in the number of antimicrobial
route of administration for different groups of antimicrobials. The vertical dashed line indicates a RR of 1 . 
where species differ in their ecology and reservoirs (De Visscher et al., 2014). Various species are likely to be exposed to low concentrations of antimicrobials in some tissues after systemic, but not necessarily intramammary, administration of antimicrobials. These bacteria, when shed to the environment, may cause IMI. An association between systemically administered cephalosporins and diminished inhibition zone diameters in NAS isolated from the udder was recently reported (Stevens et al., 2018). In contrast, associations between AMU and AMR irrespective of route of administration were observed for $S$. aureus isolated from the udder (Saini et al., 2012b), a well-known pathogen that is not opportunistic as some NAS species. In that regard, the opportunistic characteristic of some NAS species, and low concentrations of antimicrobials achieved in specific body sites (including the udder) following systemic administration of antimicrobials, are the most plausible factors related to our findings.

Antimicrobial molecules were usually administered by a single route. For penams (e.g., penicillins), which were administered by 2 distinct routes, the semisynthetic group $3 \beta$-lactamase-resistant cloxacillin was commonly administered intramammarily, whereas the group 1 penicillin $\mathrm{G}$ procaine was commonly administered parenterally. Therefore, despite being grouped into a single class, penicillin types should be considered distinct molecules that may yield different AMR profiles for the same isolates in vitro (Kimberlin et al., 1978; Turutoglu et al., 2006). Hence, it is virtually impossible to discern molecule and route effects when studying associations between AMU and AMR in NAS. Associations could therefore either be a consequence of route of administration or molecule of choice. Still, it is possible and even likely that observed effects were a result of both factors. When looking at route-specific associations, findings were consistent in terms of directionality, irrespective of systemic antimicrobial administered, and nondetected associations were probably a consequence of low power. In terms of antimicrobials, results from third-generation cephalosporins, where ceftiofur was the most commonly used molecule irrespective of route, had the same type of consistency. Similar findings were present for macrolides, where erythromycin was the most commonly used molecule, irrespective of route. The 4 herds that recorded at least 1 ADD per cow-year using macrolides intramammarily did not record any systemic therapy using the same drug class, and estimated prevalence of macrolide resistance in these herds was 0.13 versus 0.08 for remaining herds (results not shown). Hence, it is very likely that use of macrolides was associated with prevalence of AMR, irrespective of route, whereas effect sizes (e.g., RR) should be conditional on the same. In addition, use of first-generation cephalosporins was not associated with prevalence of $\beta$-lactam resistance, which might explain why these antimicrobials have been used as successfully as third-generation cephalosporins for bacteriological cure of CM caused by gram-positive bacteria (Schukken et al., 2013).

An association between AMU and presence of ARG is well established (Harvey et al., 2009; Looft et al., 2012; Sandberg and LaPara, 2016). In the present study, systemic use of macrolides and third-generation cephalosporins was associated with prevalence of erm and $b l a_{\mathrm{ARL}}$ genes in NAS, respectively. The $b l a_{\mathrm{ARL}}$ gene was recently described as being associated with penicillin resistance in $S$. arlettae isolated from bovine mastitis, and depending on expression levels, it can increase the MIC against cephalosporins (Andreis et al., 2017).

Table 3. Relative risk of presence of antimicrobial resistance genes in non-aureus staphylococci by antimicrobial used and route of administration

\begin{tabular}{|c|c|c|c|c|c|c|c|}
\hline \multirow{2}{*}{ Antimicrobial } & \multirow{2}{*}{ Gene } & \multicolumn{4}{|c|}{ Route of administration } & & \\
\hline & & \multicolumn{2}{|c|}{ Intramammary } & \multicolumn{2}{|c|}{ Systemic } & \multicolumn{2}{|c|}{ All } \\
\hline Penicillins & blaZ & 1.66 & $0.52-5.34$ & 1.66 & $0.80-3.46$ & 1.66 & $0.87-3.16$ \\
\hline Penicillin combinations & blaZ & 1.14 & $0.87-1.48$ & - & - & 1.14 & $0.87-1.48$ \\
\hline Third-generation cephalosporins & blaZ & 0.01 & $0-9.78$ & 1.34 & $0.90-2.00$ & 1.26 & $0.83-1.89$ \\
\hline Penicillin combinations & $b l a_{\mathrm{ARL}}$ & 1.21 & $0.84-1.74$ & - & - & 1.21 & $0.84-1.74$ \\
\hline Third-generation cephalosporins & $b l a_{\mathrm{ARL}}$ & 0.92 & $0-100+$ & 1.95 & $1.08-3.52$ & 1.92 & $1.07-3.47$ \\
\hline First-generation cephalosporins & $b l a_{\mathrm{ARL}}$ & 0.59 & $0.11-3.05$ & - & - & 0.65 & $0.13-3.17$ \\
\hline Macrolides & $\mathrm{erm}$ & - & - & 10.63 & $2.79-40.59$ & 10.61 & $2.75-40.97$ \\
\hline Macrolides & $m p h C$ & - & - & 1.08 & $0.49-2.38$ & 1.12 & $0.51-2.46$ \\
\hline Macrolides & $m s r A$ & - & - & 0.71 & $0.17-2.93$ & 0.73 & $0.18-2.98$ \\
\hline Tetracyclines & tet & - & - & 2.41 & $1.42-4.08$ & 2.40 & $1.42-4.08$ \\
\hline
\end{tabular}

${ }^{1}$ Relative risk (RR) per unit increase in antimicrobial daily dose per cow-year.

${ }^{2} 95 \%$ CI of RR. 
Systemic use of third-generation cephalosporins was either followed or preceded by an increased prevalence of $S$. arlettae harboring this genetic determinant. The cross-sectional nature of our study limited a conclusive assessment of the causality our findings; however, this result was alarming, especially because $S$. arlettae is the NAS species with highest prevalence of AMR isolated from dairy cows (Nobrega et al., 2018). Similarly, prevalence of tet genes in NAS was higher for herds that used more tetracyclines systemically, despite the lack of association between systemic use of tetracyclines and drug-specific phenotypic resistance. These findings could be a consequence of 2 distinct factors. First, resistance is usually mobilized by plasmids containing several ARG (Argudin et al., 2014; Liu et al., 2017) where use of antimicrobials other than tetracyclines may select for tetracycline resistance elements. Second, unlike phenotypic methods based on clinical breakpoints that require an increase in MIC above a threshold to define resistance, presence of genetic determinants of AMR reflects acquisition of a mechanism that may either be sufficient to cause resistance or simply enhance bacteria survival in the presence of low concentrations of antimicrobials (Roychoudhury et al., 2001). Moreover, expression of genes in vivo is likely to be different than in vitro. Consequently, presence of some tet elements may not be, by itself, enough for classifying isolates as clinically resistant, in agreement with previous reports (Nobrega et al., 2018).

Blanket DCT has been 1 of the 5 points in contagious mastitis control plans for several decades (Neave et al., 1969). Despite blanket DCT being used for many years, AMR among mastitis pathogens has not increased (Oliver and Murinda, 2012). Recent results indicated that herds under selective DCT had reduced use of intramammary antimicrobials without the need for a compensatory increase in CM treatments in the following lactation (Scherpenzeel et al., 2016). According to our results, it is expected that herds using selective and blanket DCT will have a similar prevalence of AMR in NAS, due to the either weak on nonexistent association between AMR and antimicrobials administered intramammarily. Following this logic, a decrease in AMR of NAS following implementation of selective DCT would be minimal in comparison to reduced use of systemic antimicrobials.

It is expected that this study will provide the impetus for further research involving a wider selection of mastitis pathogens and antimicrobials. To better interpret our findings, we must acknowledge the limitations of our study. First, associations presented in this study are NAS-specific, and therefore cannot be generalized to other bacterial species that are commonly involved in IMI such as S. aureus, Streptococcus spp., and gram-negative pathogens. Importantly, our NASspecific results were driven by the most prevalent NAS species (i.e., S. chromogenes, S. simulans, and S. xylosus). If associations are species-specific, our estimates represent marginal effects not applicable to all species. Similarly, if associations between AMU and AMR are dependent on bacterial shedding, this study could have failed to detect true associations because a high $\mathrm{cfu} /$ $\mathrm{mL}$ threshold was used to define IMI. A second caveat arises from collapsing categories of MIC values into a binary outcome, which results in a substantial loss of information. Use of the actual MIC obtained in a wide range of tested values instead of clinical breakpoints is recommended for further assessments. Third, selection of isolates to be sequenced favored inclusion of a wide range of NAS species instead of a random selection of isolates. Hence, identified genetic associations could be biased conditional on individual species effects. Fourth, absence of associations between AMU and AMR should be interpreted carefully, as they are probably a consequence of low power. Indeed, this study had only 35 and $60 \%$ power to detect RR of 1.2 and 1.3 , respectively (results not shown). A fifth limitation is that absence of (1) sufficient number of herds using specific antimicrobials (macrolides, phenicols); and (2) sufficient number of outcomes ( $\mathrm{erm}, \mathrm{mec} A$, fexA genes as well as AMR against combination containing sulfonamides) prevented further analyses or affected precision of our estimates. Finally, although our samples were derived from a longitudinal study (Reyher et al., 2011), we provided a cross-sectional assessment of the relationship between AMR and AMU. As such, causal inferences were far less robust than the same obtained from longitudinal studies.

\section{CONCLUSIONS}

An association between AMR in NAS and AMU was present when penicillins, third-generation cephalosporins, or macrolides were administered systemically. Intrauterine and intramammary use of antimicrobials were not associated with AMR in NAS. Systemic use of macrolides, third-generation cephalosporins, and tetracyclines, drug classes of critical importance for human medicine, were associated with increased prevalence of $e r m, b l a_{\mathrm{ARL}}$, and tet genes, respectively, in NAS.

\section{ACKNOWLEDGMENTS}

This work was partially funded through the Natural Sciences and Engineering Research Council (NSERC) Industrial Research Chair in Infectious Diseases of Dairy Cattle. This project was also part of the Canadian Bovine Mastitis and Milk Quality Research 
Network program, funded by Dairy Farmers of Canada and Agriculture and Agri-Food Canada through the Dairy Research Cluster 2 Program. DBN was supported by NSERC-CREATE in Milk Quality, Alberta Innovates-Technology Futures (Alberta, Canada), and the Izaak Walton Killam Memorial scholarship (Alberta, Canada). The authors thank all dairy producers, animal health technicians, and Canadian Bovine Mastitis Research Network (CBMRN) regional coordinators (Trevor De Vries, University of Guelph; Jean-Philippe Roy and Luc Des Côteaux, University of Montreal; Kristen Reyher, University of Prince Edward Island; and Herman Barkema, University of Calgary) that participated in data collection. The CBMRN data collections were financed by the Natural Sciences and Engineering Research Council of Canada (Ottawa, ON, Canada), Alberta Milk (Edmonton, AB, Canada), Dairy Farmers of New Brunswick (Sussex, New Brunswick, Canada), Dairy Farmers of Nova Scotia (Lower Truro, NS, Canada), Dairy Farmers of Ontario (Mississauga, ON, Canada), Dairy Farmers of Prince Edward Island (Charlottetown, PE, Canada), Novalait Inc. (Québec City, QC, Canada), Dairy Farmers of Canada (Ottawa, ON, Canada), Canadian Dairy Network (Guelph, ON, Canada), Agriculture and Agri-Food Canada (Ottawa, ON, Canada), Public Health Agency of Canada (Ottawa, ON, Canada), Technology PEI Inc. (Charlottetown, PE, Canada), Université de Montréal (Montréal, QC, Canada), and University of Prince Edward Island (Charlottetown, PE, Canada) through the CBMQRN (Saint-Hyacinthe, QC, Canada).

\section{REFERENCES}

Andreis, S. N., V. Perreten, and S. Schwendener. 2017. Novel betaLactamase blaARL in Staphylococcus arlettae. MSphere 2:e0117e17.

Argudin, M. A., M. C. Mendoza, M. C. Martin, and M. R. Rodicio. 2014. Molecular basis of antimicrobial drug resistance in Staphylococcus aureus isolates recovered from young healthy carriers in Spain. Microb. Pathog. 74:8-14.

Barkema, H. W., M. A. von Keyserlingk, J. P. Kastelic, T. J. Lam, C. Luby, J. P. Roy, S. J. LeBlanc, G. P. Keefe, and D. F. Kelton. 2015. Invited review: Changes in the dairy industry affecting dairy cattle health and welfare. J. Dairy Sci. 98:7426-7445.

Benjamini, Y., and Y. Hochberg. 1995. Controlling the false discovery rate: A practical and powerful approach to multiple testing. J. R. Stat. Soc. B 57:289-300.

Blondeau, J. M. 2009. New concepts in antimicrobial susceptibility testing: The mutant prevention concentration and mutant selection window approach. Vet. Dermatol. 20:383-396.

Clinical and Laboratory Standards Institute. 2013. Performance standards for antimicrobial disk and dilution susceptibility tests for bacteria isolated from animals; approved standard-Fourth edition. Clinical and Laboratory Standards Institute, Wayne, PA.

Clinical and Laboratory Standards Institute. 2016. Performance standards for antimicrobial susceptibility testing. 26th ed. Clinical and Laboratory Standards Institute, Wayne, PA.

Condas, L. A. Z., J. De Buck, D. B. Nobrega, D. A. Carson, S. Naushad, S. De Vliegher, R. N. Zadoks, J. R. Middleton, S. Dufour, J.
P. Kastelic, and H. W. Barkema. 2017. Prevalence of non-aureus staphylococci species causing intramammary infections in Canadian dairy herds. J. Dairy Sci. 100:5592-5612.

Cribari-Neto, F., and A. Zeileis. 2010. Beta regression in R. J. Stat. Softw. 34:1-24.

Davies, J., and D. Davies. 2010. Origins and evolution of antibiotic resistance. Microbiol. Mol. Biol. Rev. 74:417-433.

De Visscher, A., S. Piepers, F. Haesebrouck, K. Supre, and S. De Vliegher. 2017. Coagulase-negative Staphylococcus species in bulk milk: Prevalence, distribution, and associated subgroup- and species-specific risk factors. J. Dairy Sci. 100:629-642.

De Visscher, A., K. Supre, F. Haesebrouck, R. N. Zadoks, V. Piessens, E. Van Coillie, S. Piepers, and S. De Vliegher. 2014. Further evidence for the existence of environmental and host-associated species of coagulase-negative staphylococci in dairy cattle. Vet. Microbiol. 172:466-474.

Erskine, R. J., P. C. Bartlett, J. L. VanLente, and C. R. Phipps. 2002. Efficacy of systemic ceftiofur as a therapy for severe clinical mastitis in dairy cattle. J. Dairy Sci. 85:2571-2575.

EUCAST (European Committee on Antimicrobial Susceptibility Testing). 2017. Breakpoint tables for interpretation of MICs and zone diameters, version 7.1. Accessed Mar. 17, 2018. http://www.eucast .org/fileadmin/src/media/PDFs/EUCAST_files/Breakpoint tables/v_7.1_Breakpoint_Tables.pdf.

Gupta, S. K., B. R. Padmanabhan, S. M. Diene, R. Lopez-Rojas, M. Kempf, L. Landraud, and J. M. Rolain. 2014. ARG-ANNOT, a new bioinformatic tool to discover antibiotic resistance genes in bacterial genomes. Antimicrob. Agents Chemother. 58:212-220.

Harvey, R., J. Funk, T. E. Wittum, and A. E. Hoet. 2009. A metagenomic approach for determining prevalence of tetracycline resistance genes in the fecal flora of conventionally raised feedlot steers and feedlot steers raised without antimicrobials. Am. J. Vet. Res. 70:198-202.

Jensen, V. F., E. Jacobsen, and F. Bager. 2004. Veterinary antimicrobial-usage statistics based on standardized measures of dosage. Prev. Vet. Med. 64:201-215.

Jia, B., A. R. Raphenya, B. Alcock, N. Waglechner, P. Guo, K. K. Tsang, B. A. Lago, B. M. Dave, S. Pereira, A. N. Sharma, S. Doshi, M. Courtot, R. Lo, L. E. Williams, J. G. Frye, T. Elsayegh, D. Sardar, E. L. Westman, A. C. Pawlowski, T. A. Johnson, F. S. Brinkman, G. D. Wright, and A. G. McArthur. 2017. CARD 2017: expansion and model-centric curation of the comprehensive antibiotic resistance database. Nucleic Acids Res. 45:D566-D573.

Jorgensen, S. B., A. Soraas, A. Sundsfjord, K. Liestol, T. M. Leegaard, and P. A. Jenum. 2017. Fecal carriage of extended spectrum betalactamase producing Escherichia coli and Klebsiella pneumoniae after urinary tract infection-A three year prospective cohort study. PLoS One 12:e0173510.

Kimberlin, C., F. Khalid, and A. Hariri. 1978. Resistance of staphylococci to penicillin-G and cloxacillin. Pahlavi Med. J. 9:182-192.

Kromker, V., and S. Leimbach. 2017. Mastitis treatment-reduction in antibiotic usage in dairy cows. Reprod. Domest. Anim. 52:21-29.

Lakin, S. M., C. Dean, N. R. Noyes, A. Dettenwanger, A. S. Ross, E. Doster, P. Rovira, Z. Abdo, K. L. Jones, J. Ruiz, K. E. Belk, P. S. Morley, and C. Boucher. 2017. MEGARes: An antimicrobial resistance database for high throughput sequencing. Nucleic Acids Res. 45:D574-D580.

Lam, T., J. Jansen, and R. J. Wessels. 2017. The RESET Mindset Model applied on decreasing antibiotic usage in dairy cattle in the Netherlands. Ir. Vet. J. 70:5.

Liu, B. H., C. W. Lei, A. Y. Zhang, Y. Pan, L. H. Kong, R. Xiang, Y. X. Wang, Y. X. Yang, and H. N. Wang. 2017. Colocation of the multiresistance gene $c f r$ and the fosfomycin resistance gene fosD on a novel plasmid in Staphylococcus arlettae from a chicken farm. Antimicrob. Agents Chemother. 61:e01388-17.

Locatelli, C., L. Scaccabarozzi, G. Pisoni, and P. Moroni. 2010. CTXM1 ESBL-producing Klebsiella pneumoniae ssp. pneumoniae isolated from cases of bovine mastitis. J. Clin. Microbiol. 48:38223823.

Looft, T., T. A. Johnson, H. K. Allen, D. O. Bayles, D. P. Alt, R. D. Stedtfeld, W. J. Sul, T. M. Stedtfeld, B. Chai, J. R. Cole, S. A. 
Hashsham, J. M. Tiedje, and T. B. Stanton. 2012. In-feed antibiotic effects on the swine intestinal microbiome. Proc. Natl. Acad. Sci. USA 109:1691-1696.

National Mastitis Council. 1999. Laboratory Handbook on Bovine Mastitis. Natl. Mastitis Counc. Inc., Madison, WI.

Naushad, S., H. W. Barkema, C. Luby, L. A. Condas, D. B. Nobrega, D. A. Carson, and J. De Buck. 2016. Comprehensive phylogenetic analysis of bovine non-aureus staphylococci species based on whole-genome sequencing. Front. Microbiol. 7:1990.

Neave, F. K., F. H. Dodd, R. G. Kingwill, and D. R. Westgarth. 1969. Control of mastitis in the dairy herd by hygiene and management. J. Dairy Sci. 52:696-707.

Nobrega, D. B., J. De Buck, S. A. Naqvi, G. Liu, S. Naushad, V. Saini, and H. W. Barkema. 2017. Comparison of treatment records and inventory of empty drug containers to quantify antimicrobial usage in dairy herds. J. Dairy Sci. 100:9736-9745.

Nobrega, D. B., S. Naushad, S. A. Naqvi, L. A. Z. Condas, V. Saini, J. P. Kastelic, C. Luby, J. De Buck, and H. W. Barkema. 2018. Prevalence and genetic basis of antimicrobial resistance in nonaureus staphylococci isolated from Canadian dairy herds. Front. Microbiol. 9:256.

O'Neill, J. 2016. Tackling drug-resistant infections globally: Final report and recommendations. The review on antimicrobial resistance. Accessed Mar. 17, 2018. https://amr-review.org/sites/ default/files/160518_Final paper_with cover.pdf.

Oliver, S. P., and S. E. Murinda. 2012. Antimicrobial resistance of mastitis pathogens. Vet. Clin. North Am. Food Anim. Pract. $28: 165-185$.

Pol, M., and P. L. Ruegg. 2007. Treatment practices and quantification of antimicrobial drug usage in conventional and organic dairy farms in Wisconsin. J. Dairy Sci. 90:249-261.

R Core Team. 2017. R: A language and environment for statistical computing. R Foundation for Statistical Computing, Vienna, Austria.

Reyher, K. K., S. Dufour, H. W. Barkema, L. Des Coteaux, T. J. Devries, I. R. Dohoo, G. P. Keefe, J. P. Roy, and D. T. Scholl 2011. The National Cohort of Dairy Farms-a data collection platform for mastitis research in Canada. J. Dairy Sci. 94:1616-1626.

Roychoudhury, S., T. L. Twinem, K. M. Makin, M. A. Nienaber, C. Li, T. W. Morris, B. Ledoussal, and C. E. Catrenich. 2001. Staphylococcus aureus mutants isolated via exposure to nonfluorinated quinolones: detection of known and unique mutations. Antimicrob. Agents Chemother. 45:3422-3426.

Saini, V., J. T. McClure, D. Leger, S. Dufour, A. G. Sheldon, D. T. Scholl, and H. W. Barkema. 2012a. Antimicrobial use on Canadian dairy farms. J. Dairy Sci. 95:1209-1221.

Saini, V., J. T. McClure, D. T. Scholl, T. J. DeVries, and H. W. Barkema. 2012b. Herd-level association between antimicrobial use and antimicrobial resistance in bovine mastitis Staphylococcus aureus isolates on Canadian dairy farms. J. Dairy Sci. 95:1921-1929.

Sandberg, K. D., and T. M. LaPara. 2016. The fate of antibiotic resistance genes and class 1 integrons following the application of swine and dairy manure to soils. FEMS Microbiol. Ecol. 92:fiw001.

Scherpenzeel, C. G. M., I. E. M. den Uijl, G. van Schaik, R. G. M. Olde Riekerink, H. Hogeveen, and T. J. G. M. Lam. 2016. Effect of different scenarios for selective dry-cow therapy on udder health, antimicrobial usage, and economics. J. Dairy Sci. 99:3753-3764.

Schukken, Y. H., M. J. Zurakowski, B. J. Rauch, B. Gross, L. L. Tikofsky, and F. L. Welcome. 2013. Noninferiority trial comparing a first-generation cephalosporin with a third-generation cephalosporin in the treatment of nonsevere clinical mastitis in dairy cows. J. Dairy Sci. 96:6763-6774.

Stevens, M., S. Piepers, K. Supre, and S. De Vliegher. 2018. Antimicrobial consumption on dairy herds and its association with antimicrobial inhibition zone diameters of non-aureus staphylococci and Staphylococcus aureus isolated from subclinical mastitis. J. Dairy Sci. 101:3311-3322.

Tang, K. L., N. P. Caffrey, D. B. Nóbrega, S. C. Cork, P. E. Ronksley, H. W. Barkema, A. J. Polachek, H. Ganshorn, N. Sharma, J. D. Kellner, and W. A. Ghali. 2017. Restricting the use of antibiotics in food-producing animals and its associations with antibiotic resistance in food-producing animals and human beings: a systematic review and meta-analysis. Lancet Planet Health 1:e316-e327.

Thorberg, B. M., I. Kuhn, F. M. Aarestrup, B. Brandstrom, P. Jonsson, and M. L. Danielsson-Tham. 2006. Pheno- and genotyping of Staphylococcus epidermidis isolated from bovine milk and human skin. Vet. Microbiol. 115:163-172.

Turutoglu, H., S. Ercelik, and D. Ozturk. 2006. Antibiotic resistance of Staphylococcus aureus and coagulase-negative staphylococci isolated from bovine mastitis. Bull. Vet. Inst. Pulawy. 50:41-45.

Venables, W. N., and B. D. Ripley. 2002. Modern Applied Statistics with S. 4th ed. Springer-Verlag, New York, NY.

World Healh Organization. 2016. Critically important antimicrobials for human medicine-5th revision. World Health Organization, Geneva, Switzerland. Accessed Mar. 17, 2018. http://www.who.int/ foodsafety/areas_work/antimicrobial-resistance/cia/en/.

Zankari, E., H. Hasman, S. Cosentino, M. Vestergaard, S. Rasmussen, O. Lund, F. M. Aarestrup, and M. V. Larsen. 2012. Identification of acquired antimicrobial resistance genes. J. Antimicrob. Chemother. 67:2640-2644

Zhang, L., Y. Huang, Y. Zhou, T. Buckley, and H. H. Wang. 2013 Antibiotic administration routes significantly influence the levels of antibiotic resistance in gut microbiota. Antimicrob. Agents Chemother. 57:3659-3666. 